\title{
Baetis bisri n. sp., Ephéméroptère nouveau du Liban (Baetidae)
}

\author{
A.G.B. Thomas 1
}

A. $\mathrm{Dia}^{2}$

Description illust rée de la larve au dernjer stade de Baefis bisri n, sp., rencontrée dans le Sud du Liban. B. bisri présente des affinités morphologiques avec les espèces du groupe rhodani et en particulier avec B. canariensis Müller-Liebenau, 1971 .

Baetis bisri sp. n., a new mayfly from Lebanon (Ephemeroptera, Baetidae).

An illustrated description of the last-instar larva of Baetis hisri sp. n. captured in the south of Lebanon. The affinities of $B$. bisri lie with the rhodani group, especially with B. canariensis Müller-Liebenau, 1971.

De nombreuses larves d'une espèce nouvelle du genre Baetis ont été récoltées lors de l'étude faunistique de plusieurs cours d'eau du Sud du Liban (Dia 1983, s. n. "sp. 3 ").

\section{1. - Baetis bisri Thomas et Dia n. sp. : description}

Larve au dernier stade.

Diagnose sommaire :

Espèce du groupe rhodani, de taille moyenne à assez grande ; coloration plutôt contrastée ; paracerque de longueur au moins égale ou supérieure à la moitié de celle des cerques.

Tête.

Insertions musculaires sur le crâne assez peu contrastées.

Antennes: le scape et le pédicelle portent des écailles plutôt longues ( $/ \mathrm{ig} .1$ ) en plus de petites soies fines.

Labre (fig. 2) : il est proche de celui de B. ilex Jacob et Zimmermann, 1981. Il est relativement long et étroit (rapport de sa largeur à sa longueur, toutes soies exclues, compris entre 1,08 et 1,24 pour 17

1. Laboratoire d'Hydrobiologie, ERA 702 du CNRS, Université Paul Sabatier, 118 route de Narbonne, 31062 Toulouse Ceder, France.

2. Département de Sciences Naturelles, Université Libanaise. Faculte des Sciences, Hadeth-Beyrouth, Liban.

Ce travail a été subventionné par le C.N.R.S. libanais. exemplaires mesurés, $\overline{\mathrm{m}}=1,15$ ). La région antérieure est saillante mais franchement entaillée dans le plan de symétrie. Nombre de grandes soies frontales : $1+7$ (assez souvent 6 ou 8 , plus rarement 5 ou 9). Ces soies sont disposées selon une ligne légèrement concave se rapprochant très progressivement du bord du labre et de ses soies marginales postérieures. Ceci entraine souvent une appréciation assez difficile de leur nombre exact et une incertitude fréquente d'une unité. D'autre part, la dernière soie est parfois remplacée par une écaille aiguë.

Mandibules : zone incisive à dents très marquées et arrondies (fig. 3). Prosthecas franchement asymétriques.

Maxilles: apex des palpes maxillaires (fig. 4) légèrement tronconique du côté interne; une très petite denticulation apicale, plutót peu visible jusqu'à un grossissement d'au moins 300 , probablement parfois absente.

Labium : glosses proches de celles de B. rhodani mais paraglosses portant à l'apex des soies plus nombreuses, réparties sur 5.6 rangs (fig. 5). Palpe labial intermédiaire entre celui de $B$. rhodani Pictet, 1843-45, plus large et plus épais, et celui de $B$. canariensis Müller-Liebenau, 1971, au contraire plus étroit et plus nettement anguleux à l'apex. $2^{\mathrm{e}} \mathrm{seg}$ ment (fig. 6) : contour interne à peu près rectiligne et décrochement apical très marqué. $3^{\mathrm{e}}$ article régulièrement arrondi jusqu'à un léger mamelon au sommet ; contours interne et externe presque symétriques, l'interne un peu plus convexe. 

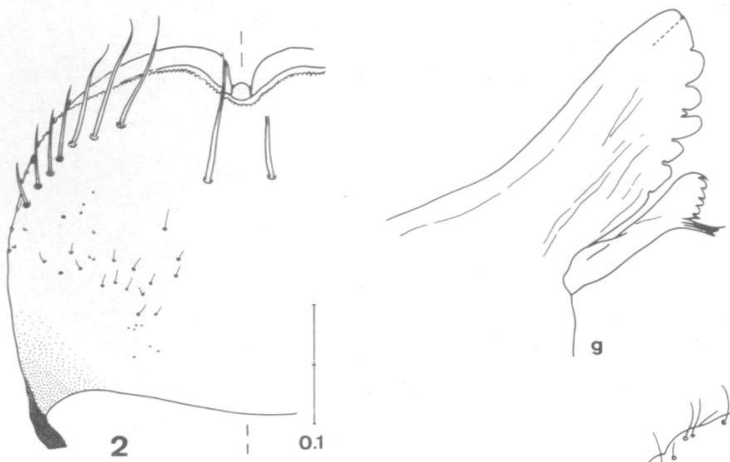

3
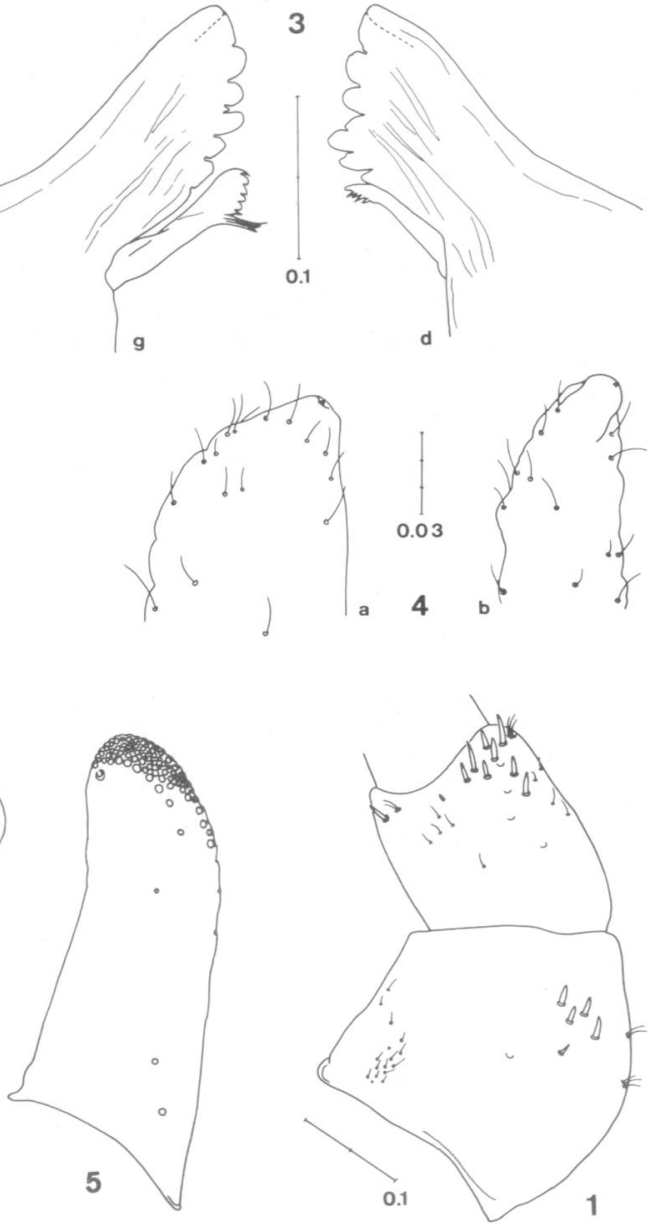

FIG. 1 à 6. Larves de Baetis bisri $\mathrm{n}$. sp. au dernier stade. Echelle en $\mathrm{mm}$.

1 : scape et pédicelle. $2:$ labre. $3:$ apex et prostheca des mandibules droite (d) et gauche $(\mathrm{g}) .4:$ apex du palpe maxillaire chez deux spécimens $\left(a\right.$ et $b$ ) d'orientation différente. 5 : paraglosse. face ventrale. $6: 2^{e}$ et $3^{e}$ articles du paipe labial avec variations des contours externe (e) et interne (i), face ventrale. 

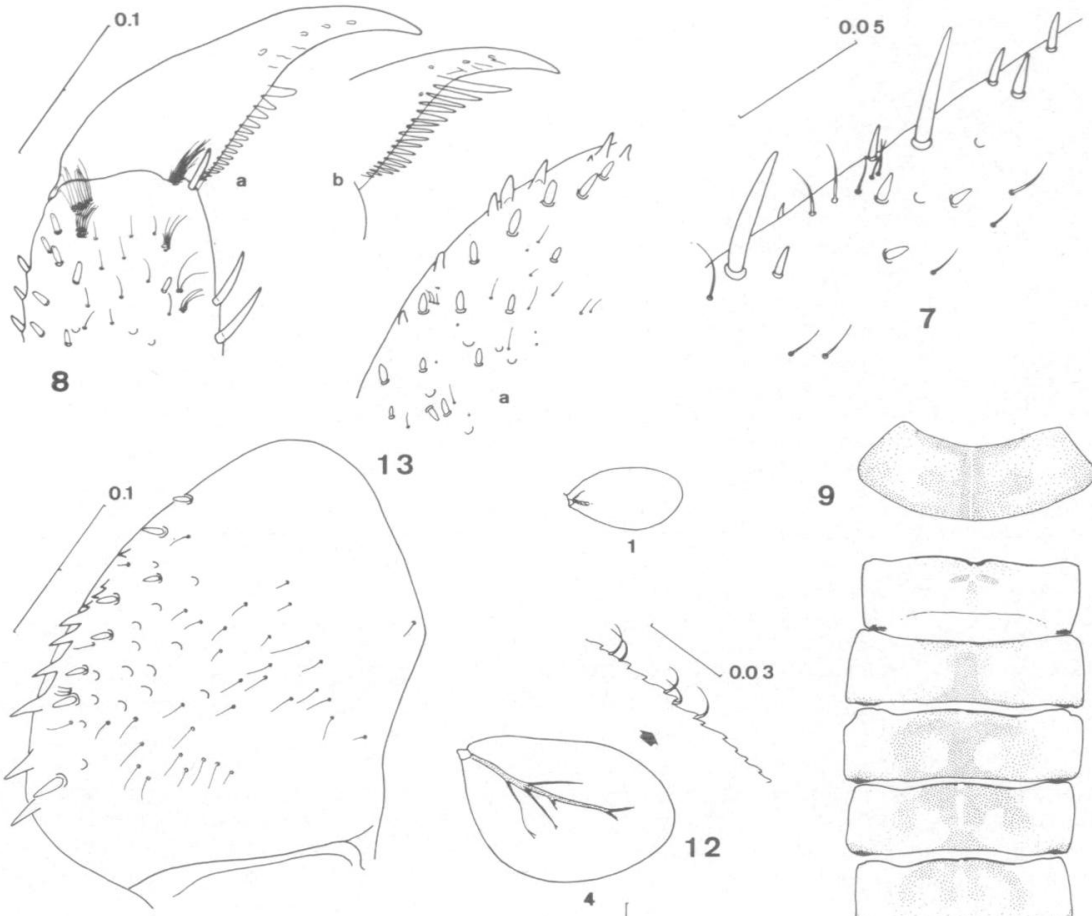

9
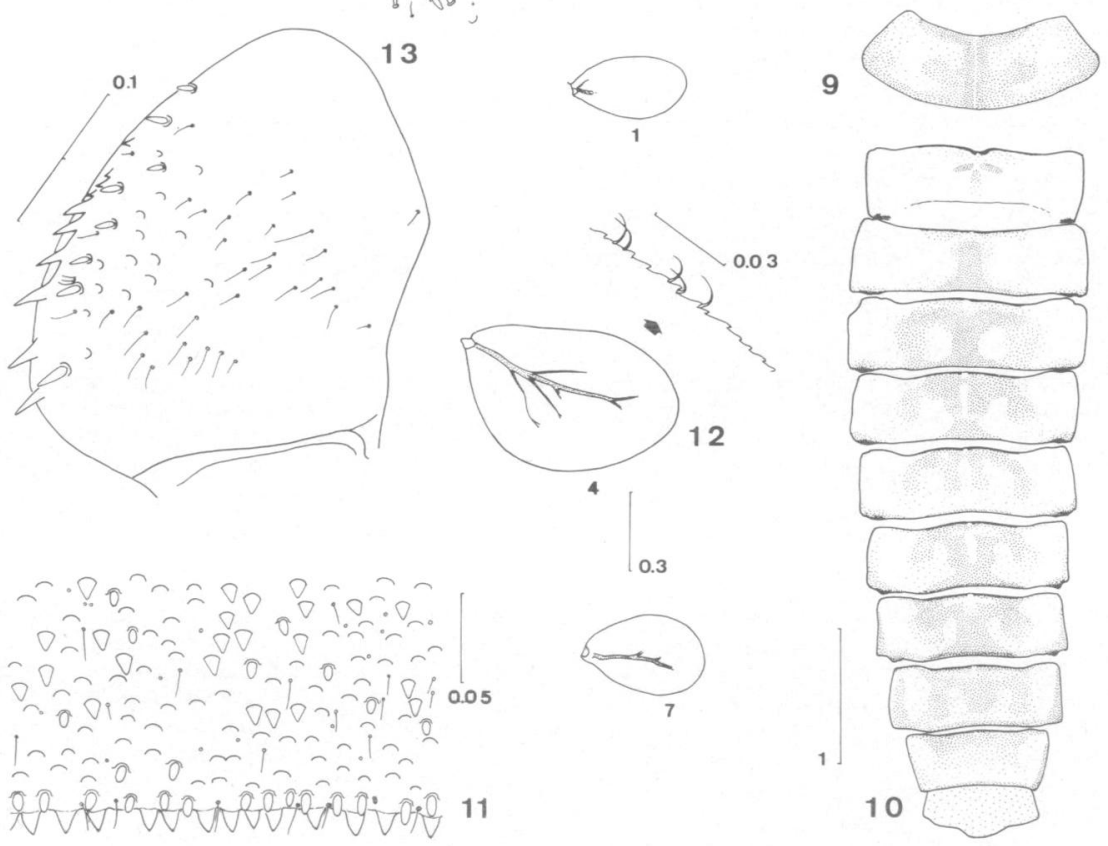

Fig 7 à 13. Larves de Baetis bisri n. sp. au dernier stade. Echelle en mm.

7 : bord postérieur du fémur, dans la région médiane. 8 : griffe tarsale (a et b : variations de la longueur des denticulations sur deux exemplaires). 9 : pronotum. 10 : coloration dorso-abdominale. 11 : ornementation cuticulaire et bord postérieur du $7^{\mathrm{e}}$ tergite abdominal. 12 : branchies droites des $1^{\text {ere }}, 4^{e}$ et $7^{e}$ paires, et bord postéro-externe de la $4^{\mathrm{e}}$, 13 et 13 a $:$ paraprocte chez deux individus. 


\section{Thorax.}

Pronotum à maculations brunes assez peu étendues, surtout visibles près du plan de sýmétrie (fig. 9).

Pattes claires, blanc jaunàtre sauf l'apex des tarses et l'articulation fémur-tibia, brun soutenu. D'au. tre part, peu avant l'émergence, deux taches préapicales brunes, très visibles, apparaissent sur les fémurs, l'une du côté interne, l'autre du côté externe : elles appartiennent à la subimago.

Région médio-dorsale du fémur couverte de petites écailles arrondies et d'impressions concaves. Bord postéro-externe porteur de fortes écailles allongées, entremêlées d'écailles plus courtes, coniques et de fines soies et impressions concaves (fig. 7).

Griffes tarsales proches de celles de $B$. rhodani: assez fortes, relativement peu recourbées et dépourvues de soies préapicales (fig. 8). Denticulations ventrales au nombre de 12 à 14 (le plus souvent 13), plus rarement 11,15 ou 16. La longueur de ces denticulations varie selon l'usure, et la première, ou les deux premières, souvent très longues, peuvent être brisées.

Abdomen.

Coloration dorsale assez contrastée, avec des maculations brunes sur fond clair, variables en étendue mais surtout visibles sur les tergites $2,3,4,6$, 7 et 8 ( $f i g .10)$.

L'ornementation superficielle des tergites est complexe avec :

- de nombreuses impressions concaves très ouvertes ;

- des formations coniques minces, larges et aiguës ;

- des soies antéro-postérieures simples, petites, assez dispersées :

- et sur la moitié distale des tergites, des écailles arrondies.

Le bord postérieur des tergites est constitué par de fortes épines, aiguës et à large base, entremêlées de petites soies; ces épines sont doublées, légèrement en retrait, par un rang sous marginal d'écail. les arrondies (fig. 11). Les tergites abdominaux sont donc proches de ceux de $B$. canariensis MüllerLiebenau, 1971.

7 paires de branchies arrondies à l'apex, larges et asymétriques avec un contour postéro-interne nettement plus saillant que le bord externe (fig. 12): système trachéen parfois pigmenté, violacé noirâtre. Le bord des branchies porte de fines denticulations, beaucoup plus petites que les protubérances écailleuses de $B$. rhodani

Paraproctes (fig. 13). Surface ornementée de quelques impressions concaves peu marquées, de fines soies simples et d'un petit nombre d'écailles facultatives ( 0 a 5 ). Bord porteur de grosses épines marginales et de fortes écailles sous marginales (en général moins d'une dizaine de chaque).

Cerques plutôt clairs, jaunâtres ; paracerque de longueur comprise entre la moitié et les trois quarts de celle des cerques $(0,51$ à 0,70 sur 13 exemplaires mesurés; $\overline{\mathrm{m}}=0,59$ ).

Taille.

L'examen des fourreaux alaires des larves montre que la période de vol s'étend pratiquement sur toute l'année. La taille des larves au dernier stade est par conséquent très variable.

Longueurs (en $\mathrm{mm}$ ) :

- du corps : 5,2 à $6,9(\sigma), 5,8$ à $8,9(8)$;

- des cerques: 3,1 à $5,3(\sigma), 3,7$ à $5,8(8)$;

- du paracerque : 1,8 à $2,7(\circ), 2,2$ à $3,4(\%)$.

\section{2. - Matériel examiné et répartition}

150 larves, dont une grande partie au dernier stade, provenant de deux bassins hydrographiques du Sud-Ouest du Liban, situés entre Saîda et Beyrouth (A. Dia leg.):

- Bassin du Nahr el Aouali :

Ruisseau d'El Barouk au pont du Jdaidet ech Choûf $(710 \mathrm{~m}$ ) 20-10-1979 (1 larve) ; source Mourched, près d'El Moukhtâra $(800 \mathrm{~m})$ 15-3-80, 10-4-80, 29-4-79, 3-6-79, 25-7-79, 15-9-79, 25-10-79 (30 l.) ; émissaire de la source Salman près de Harêt Jandal $(800 \mathrm{~m})$ 19-1-80, 3-6-79, 17-11-79 (15 l.); source Abou Kharma (850 m) 15-3-80, 29-4-79, 3-6-79, 16-8-79 (75 I.); aval de la confluence des ruisseaux Aazibi et Jezzîne $(690$ m) 16-3-80 (2 l.); r. d'El Barouk au Pont Bisri ( 380 m) 22-4-79 (1 l.), en amont de la centrale hydroélectrique de l'Aouali $(230 \mathrm{~m})$ 30-1-80 (1 l.) et en amont de celle de Joun $(50 \mathrm{~m})$ 24-12-79 (1 l); source de Joun (36 m) 22-6-80 (2 l.).

- Bassin du Nahr ed Damour :

Rivière ed Damour au pont d'El Quâdi $(260 \mathrm{~m})$ 25-5-80, 31-5-80, 13-7-80, 22-8-80 (11 l. dont I'holotype : 11. \& du 25-5) ; ruisseau temporaire d'El Ghaboun $(260 \mathrm{~m}) 29.11 .80$ (5 l.) ; ruisseau d'El Hammam (45 
m) 31-5-80, 26-6-80, 29-11-80, (4 l.) ; rivière ed Damour en aval du contluent avec le ruisseau d'El Hammam (40 m) 21-2-80, 25-5-80 (2 l.).

L'espece est nommée du nom de la première station où elle a été rencontrée.

Les spécimens sont conservés en alcool à $70^{\circ} .18$ larves ont été disséquées et partiellement montées au Baume du Canada.

\section{3. - Ecologie sommaire}

Dans les réseaux hydrographiques considérés, $B$. bisri est une espèce de basse et moyenne altitude, plus caractéristique du rhithron que du crénon. Les larves ont été surtout récoltees sur substrat grossier, encroûté, à des stations où la température de l'eau ne dépasse jamais $24^{\circ} \mathrm{C}$. Les vitesses de cou. rant étaient comprises entre 10 et $80 \mathrm{~cm} / \mathrm{s}$.

Rappelons d'autre part les découvertes récentes de deux espèces du groupe rhodani dans le Caucase et en Transcaucasie : B. baksan Soldán (Soldán 1977,
Zimmermann 1981) et B. ilex Jacob et Zimmermann (Jacob et Zimmermann 1978, Zimmermann 1981) et aussi d'une troisième en Crimée : $B$. braaschi Zimmermann 1980.

\section{Travaux cltes}

Dia (A.). 1983. - Recherches sur l'écologie et la biogéographie des cuurs d'eau du Liban méridional. These de Doctorat d'Etat. Univ. Aix-Marseille III. 302 p.

Jacub (U.) et Zimmermann (W.). 1978. Eine neue Baesis- Art der rhodani-Gruppe vom Kaukasus-Baetis ilex n. sp. (Ephemeroptera, Baetidae. Em. Nachr, $22(6): 81 \cdot 88$.

Muiller-Liebenau (L.) 1969.- Revision det europaischen Arten der Gat tung Baetis Leach, 1815 (insecta, Ephemeroptera). Gewäss. Abwäss., 48 - 49:1 - 214 .

Müller-Liebenau (I.). 1971.- Ephemeroptera (Insecta) von den Kanarischen Inseln. Gewäss. Abwäss., 50 - 51: 7-40.

Soldan (T.). 1977. - Baetis baksan sp. n., a new species of maylly (Ephemeroptera, Bactidae) from Cent ral Caucasus. Acta. ent. bohemoslov., $74(4): 229 \cdot 231+1 \mathrm{pl}$.

Zimmermann (W.). 1980 - Baefis braaschi n.sp., ein bisher unbekannter Vertreter der rhodani-Gruppe von der Krim (UdSSR) (Ephemeroptera. Baetidac). Reichenbachia. 18 (28): 199 + 202.

Zimmermann (W.) 1981. - Beitrag zur Kenntnis der Gattung Baetis Leach, 1815 (Insecta, Ephemeroptera) im Kaukasus und in Transkaukasien (UdSSR). Ent. Nachr., $25(7 / 8): 97-112$. 